Case Report

\title{
Ameloblastic Odontoma with Amyloid Deposition in a Mouse
}

\author{
Minoru Ando ${ }^{1}$, Shoichi Kado ${ }^{1}$, Kana Hashimoto ${ }^{1}$, Yuriko Nagata ${ }^{1}$, Shin Iwata ${ }^{1}$, \\ Masatoshi Takahashi ${ }^{1}$, Kazumi Uchida ${ }^{1}$, and Masaharu Onoue ${ }^{1}$ \\ ${ }^{1}$ Yakult Central Institute for Microbiological Research, 1796 Yaho, Kunitachi-shi, Tokyo 186-8650, Japan
}

\begin{abstract}
A female BALB/c:Slc mouse was administered two intravenous injections of doxorubicin $(5 \mathrm{mg} / \mathrm{kg})$ at the ages of 22 and 24 weeks. When this mouse was sacrificed at the age of 30 weeks, a tumor was found in the left mandible. The tumor was composed of dental tissues at varying stages of differentiation, ranging from primitive tooth germ-like structures to mature tooth-like tissue. Amyloid deposition was noted in a part of the tumor. Based on these histopathological findings, the tumor was diagnosed as an ameloblastic odontoma with amyloid deposition.
\end{abstract}

(J Toxicol Pathol 2005; 18: 117-120)

Key words: ameloblastic odontoma, amyloid, odontogenic tumor, mouse

Few reports of odontogenic tumors in mice have been published to date. To our knowledge, only two ICR mice with spontaneous odontogenic tumors ${ }^{1,2}$ and a few transgenic and virus-infected mice with experimental odontogenic tumors ${ }^{3,4}$ have been reported. Amyloid deposition in spontaneously occurring tumors of mice has been reported in myeloma, mammary carcinoma, etc. ${ }^{5,6}$. However, no report of amyloid deposition in odontogenic tumors of mice has been published. We recently encountered a mouse in which amyloid deposition was noted in an ameloblastic odontoma, a seemingly spontaneously occurring tumor, following administration of doxorubicin. The features of this tumor are reported in this paper.

The tumor was found in the left mandibular region involving the left molars of a female BALB/c:Slc mouse sacrificed at the age of 30 weeks. The mouse had received two intravenous injections of doxorubicin (Kyowa Hakko Kogyo Co., Ltd., Tokyo, Japan) at $5 \mathrm{mg} / \mathrm{kg}$, at the ages of 22 and 24 weeks.

The mouse's head, including the tumor, was fixed in $10 \%$ neutral bufferd formalin and was decalcified with Morse fluid. It was embedded in paraffin by the conventional method. Thin sections (4-6 $\mu \mathrm{m}$ thick) were then prepared and subjected to hematoxylineosin (H-E) staining, Congo-red staining (C-R) and immunohistochemical staining (labeled streptoavidin-biotin

Received: 28 February 2005, Accepted: 13 May 2005

Mailing address: Minoru Ando, Yakult Central Institute for

Microbiological Research, 1796 Yaho, Kunitachi-shi, Tokyo

186-8650, Japan

TEL: 81-42-577-8960 FAX: 81-42-577-3020

E-mail: minoru-ando@yakult.co.jp method) using rabbit anti-rat laminin polyclonal antibody, rabbit anti-bovine keratin polyclonal antibody and mouse anti-vimentin monoclonal antibody (DakoCytomation Co., Ltd., Kyoto, Japan). The non-decalcified lower end of the tumor was immunostained with vimentin.

The tumor measured approximately $10 \times 5 \times 10 \mathrm{~mm}$ in size. It was grayish-white in color on cut surface, and was poorly demarcated from the surrounding muscular tissues (Fig. 1). Histologically, the tumor cells had grown and infiltrated the mandibular tissue, destroying the mandible. The tumor tissue was composed of an area rich in cellular components and an area rich in eosinophilic matrix (Fig. 2). In the area rich in cellular components, a primitive tooth germ-like structure was observed in the stroma composed of fibroblasts and odontoblasts. This structure showed bursiform and was bounded by a laminin-positive basal lamina. A row of keratin-positive enamel epithelial cells was seen internal to the basal lamina, and a row of vimentinpositive odontoblasts with scant cytoplasm was observed external to the basal lamina (Fig. 3). In some of structures, stellate reticulums were found near enamel epithelia.

The area of the tumor lacking a basal lamina was found to contain collagen fibers (dentin-like matrix) connecting the odontoblasts to the enamel epithelium. In the area primarily composed of an eosinophilic matrix, we noted immature dental hard tissue (composed of dentin-like matrix which was weakly eosinophilic and fibrous or was uniformly amorphous, and of an enamel-like matrix which was strongly eosinophilic and uniformly amorphous, partially assuming an oil droplet-like shape) and mature dental tissue with non-homogeneously stained material, weakly eosinophilic to basophilic. The non-homogeneously stained 


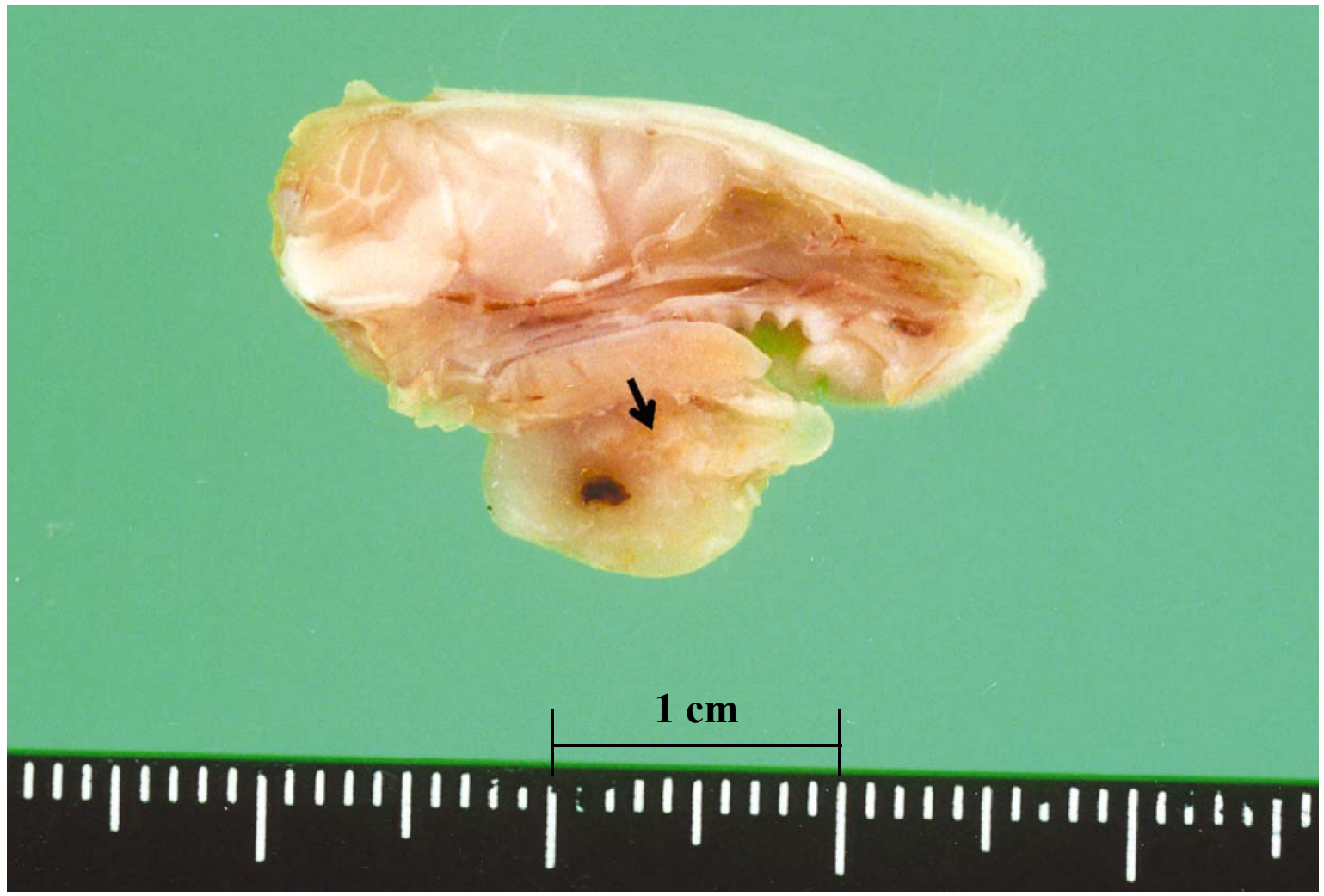

Fig. 1. Macroscopic appearance of the mass: Approximately $10 \times 5 \times 10 \mathrm{~mm}$ in size, grayish-white mass located in the left mandibular region (arrow). The mass was poorly demarcated from the surrounding muscular tissues. $\times 3.5$.

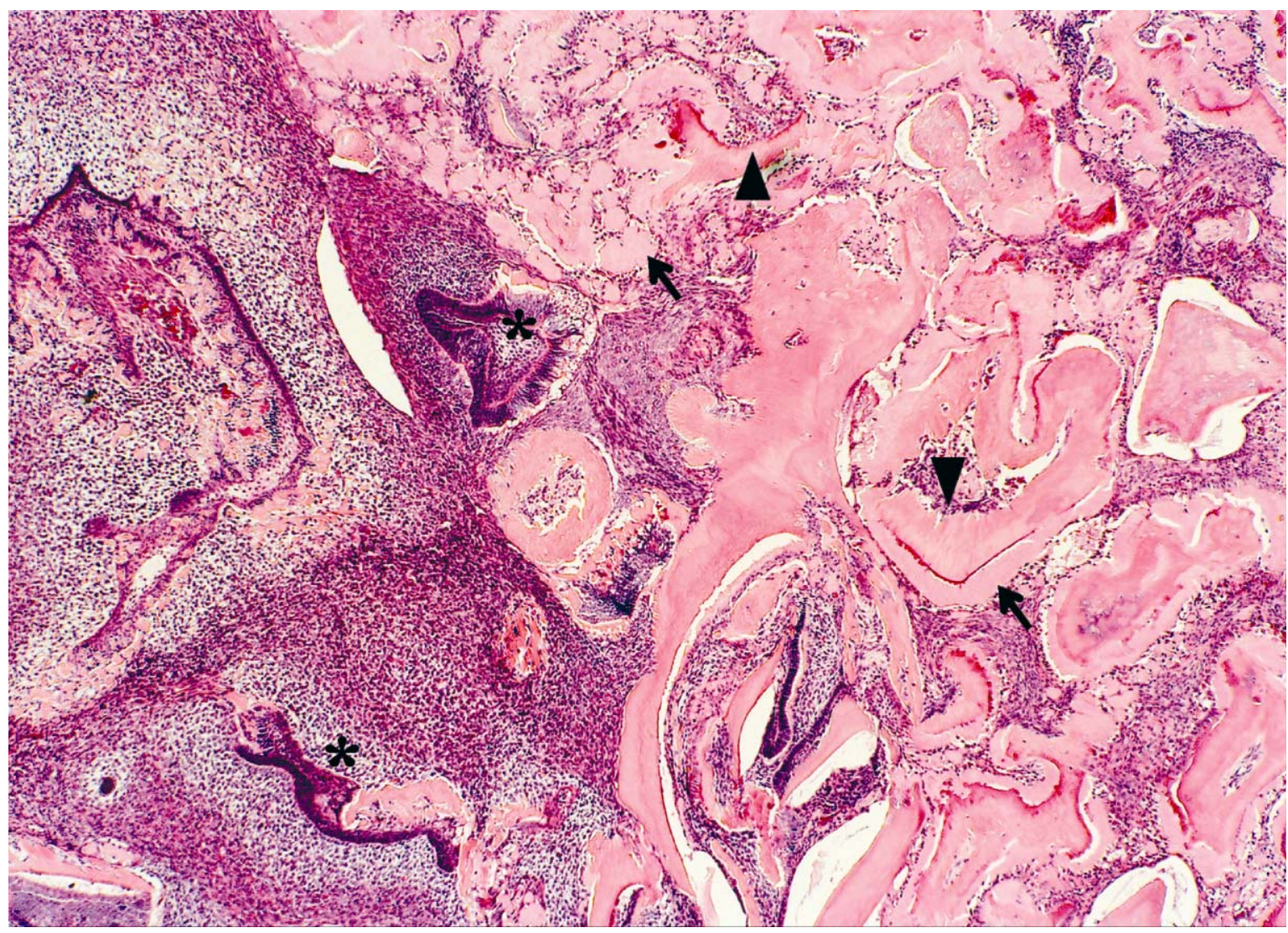

Fig. 2. Microscopic appearance: The tumor contained a primitive tooth germ-like structure $(*)$, immature dental hard tissue (arrow heads) and structures showing weak eosinophilic to basophilic staining (arrows). $\times 30$. 
a

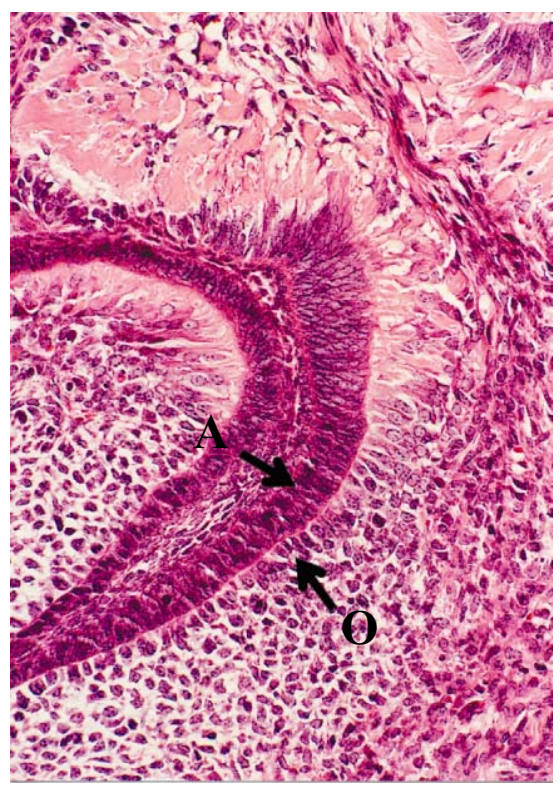

b

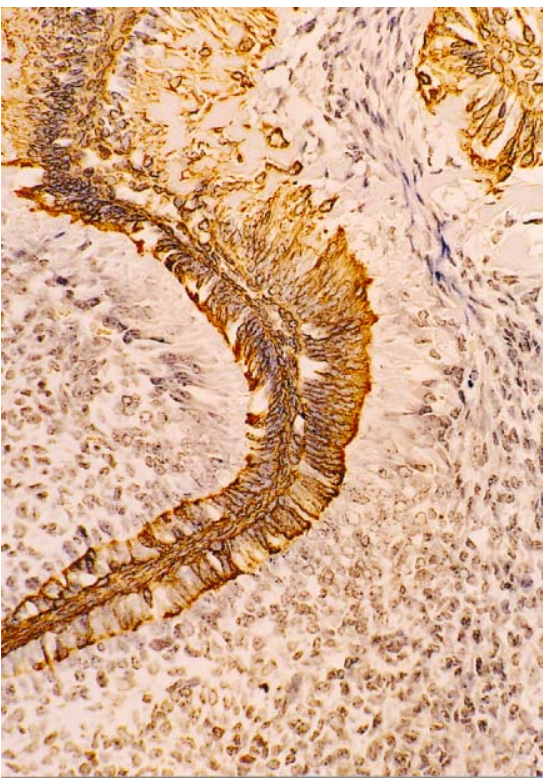

c

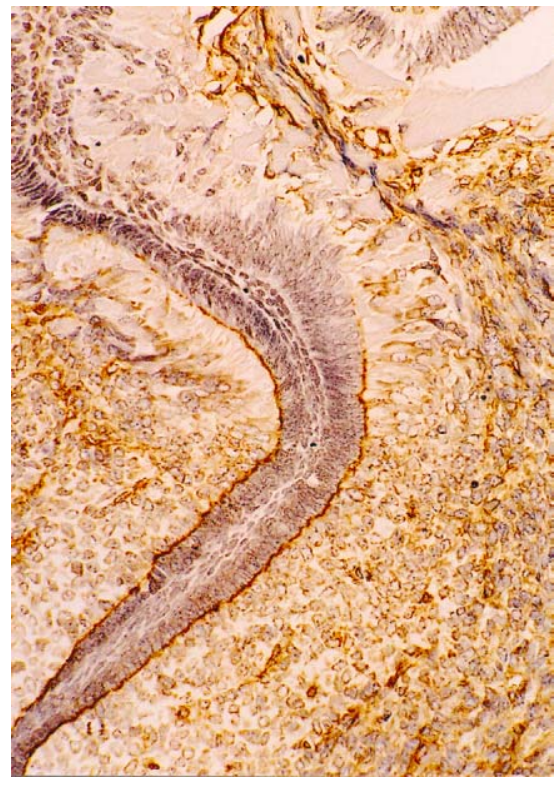

Fig. 3. A bursiform structure bounded by a basal lamina, which was lined with ameloblasts (A) on its internal aspect and odontoblasts (O) on its external aspect. Collagen fibers (dentin-like matrix) secreted by odontoblasts were observed where no basal lamina was present. $\times 85$.

a: H-E staining, b: Immunohistochemical staining with anti-bovine keratin, c: Immunohistochemical staining with anti-rat laminin.

a

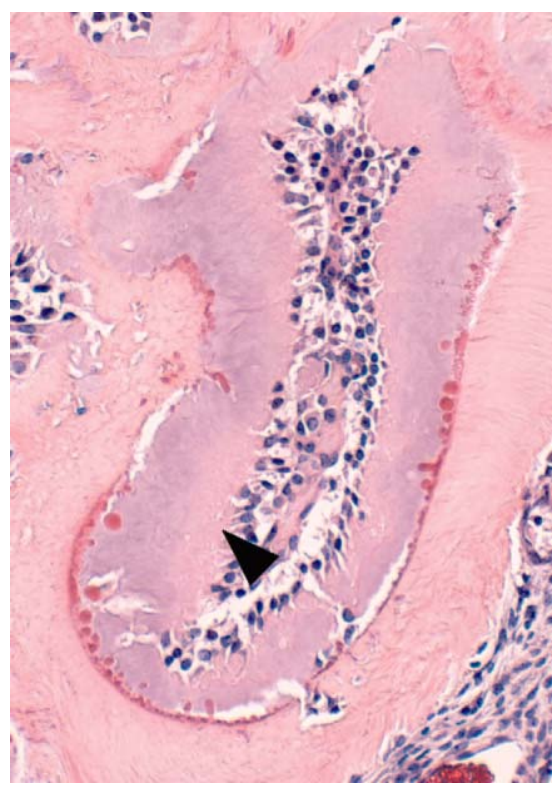

b

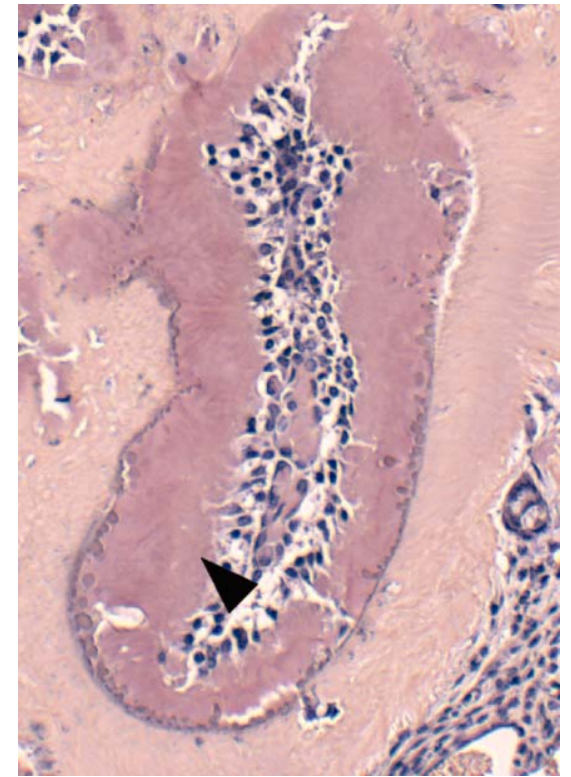

c

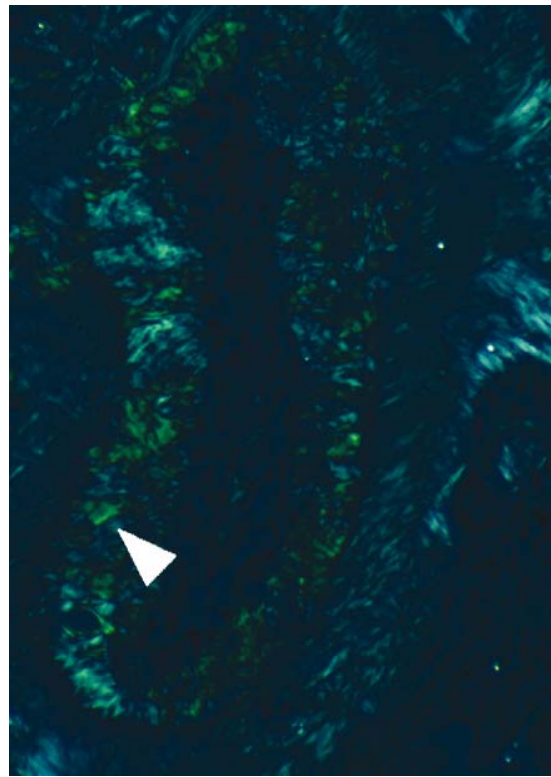

Fig. 4. The amyloid-like materials (arrow head, a) showing weak eosinophilic to basophilic appearance on H-E staining were congophilic (arrow head, b) and showed apple green birefringence under a polarized microscope (arrow head, c). $\times 96$. a: H-E staining, b: C-R staining, c: C-R staining under a polarized microscope.

slightly eosinophilic to basophilic material showed positive staining with C-R staining and yellow to green polarization under a polarized microscope, suggesting that it was composed of amyloid (Fig. 4). This material was surrounded by keratin-positive polygonal cells, some of which showed signs of transition to enamel epithelial cells. The polygonal cells thus seemed to have originated from the enamel epithelial cells.

Ameloblastic-odontomas of mice are defined as tumors found in dental tissues at varying stages from differentiation 
of primitive tooth germ-like structures to mature dental hard tissue and also in proliferation of epithelial components without the formation of dental hard tissue. Ameloblastic fibro-odontomas are defined as tumors found in dental papilla-like components and not in the stellate reticulum near the enamel epithelia. On the basis of our findings, this case was diagnosed as an ameloblastic odontoma at varying stages of dental tissues differentiation and proliferation of epithelial components without the formation of dental hard tissue. Amyloid deposition in odontogenic tumors has been reported in amyloid-producing odontogenic tumors $(\mathrm{APOT})^{7,8}$ and calcifying epithelial odontogenic tumors $(\mathrm{CEOT})^{9-12}$ in dogs and cats. In these tumors, amyloid deposition was associated with some other characteristic features (proliferating squamous epithelial-like cells arranged in strands or nests of within the fibrous stroma and the appearance of ghost cells). In the case of the mouse presented here, no proliferating squamous epithelial-like cells and ghost cells were observed within the fibrous stroma, suggesting that the tumor was not an APOT or CEOT. The tumor was finally diagnosed as an ameloblastic odontoma with amyloid deposition.

No report on odontogenic tumors caused by doxorubicin administration has been published to date. Only dysplasia of the teeth due to doxorubicin administration has been reported ${ }^{13}$. Regarding doxorubicin-associated carcinogenesis in general, induction of mammary tumors, etc., by this drug has been reported in rodents ${ }^{14}$. In these cases, however, doxorubicin had been administered for about 50 weeks to the rodents before the appearance of the tumor, up to a cumulative dose of $10 \mathrm{mg} / \mathrm{kg}$ (five injections at $2 \mathrm{mg} / \mathrm{kg}$ ). The mouse reported here had received only two doses of doxorubicin at $5 \mathrm{mg} / \mathrm{kg}$ before the appearance of the tumor in the mandible. Since the interval from the dosing to the tumor appearance was short, and no odontogenic tumor of mice associated with doxorubicin treatment has been reported until now, the involvement of doxorubicin in the onset of the tumor in this case would seem to be quite unlikely.

\section{References}

1. Nyska A, Waner T, Tal H, and Dayan D. Spontaneous ameloblastic fibro-odontoma in a female mouse. J Oral
Pathol Med. 20: 250-252. 1991.

2. Dayan D, Waner T, Harmelin A, and Nyska A. Bilateral complex odontoma in a Swiss (CD-1) male mouse. Lab Anim. 28: 90-92. 1994.

3. Gibson CW, Lally E, Herold RC, Decker S, Brinster RL, and Sandgren EP. Odontogenic tumors in mice carrying albumin-myc and albumin-ras transgens. Calcif Tissue Int. 51: 162-167. 1992.

4. Gollard RP, Slavkin HC, and Snead ML. Polyoma virusinduced murine odontogenic tumors. Oral Surg Oral Med Oral Pathol. 74: 761-767. 1992.

5. Baumal R, Wilson B, and Pass E. Experimental murine amyloidosis: a model system for studying amyloid formation. Can Med Assoc J. 113: 512-516. 1975.

6. Madi K, De Paola D, Duarte F, Takyia C, and Lima RJ. Spontaneous amyloidosis in mice with malignant neoplasms. Exp Pathol. 38: 129-134. 1990.

7. Gardner DG, Dubielzig RR, and McGee EV. The so-called calcifying epithelial odontogenic tumor in dogs and cats (amyloid-producing odontogenic tumour). J Comp Pathol. 111: 221-230. 1994.

8. Kuwamura M, Kanehara T, Yamate J, Shimada T, and Kotani T. Amyloid producing odontogenic tumor in a ShihTzu dog. J Vet Med Sci. 62: 655-657. 2000.

9. Walsh KM, Denholm LJ, and Cooper BJ. Epithelial odontogenic tumours in domestic animals. J Comp Pathol. 97: 503-521. 1987.

10. Breuer W, Geisel O, Linke RP, and Hermanns W. Light microscopic, ultrastructual, and immunohistochemical examinations of two calcifying epithelial odontogenic tumors (CEOT) in a dog and a cat. Vet Pathol. 31: 415-420. 1994.

11. Nakanishi M, Kuwamura M, Yamate J, Aoki M, and Uchida T. Calcifying epitherial odontogenic tumor in a dog. J Toxicol Pathol. 15: 171-173. 2002.

12. Ohmachi T, Taniyama H, Nakade T, Kaji Y, and Furuoka H. Calcifying epithelial odontogenic tumours in small domesticated carnivores: Histological, immunohistochemical and electron microscopical studies. J Comp Pathol. 114: 305-314. 1996.

13. Satoh H, Uesugi Y, Kawabata T, Mori K, Fujii F, Kashimoto Y, Kajimura T, and Furuhama K. Morphological classification of dental lesions induced by various antitumor drugs in mice. Toxicol Pathol. 29: 292-299. 2001.

14. Jang JJ, Takahashi M, Hasegawa R, Furukawa F, Toyoda K, Sato H, Miyakawa Y, and Hayashi Y. Mammary and renal tumor induction by low doses of adriamycin in SpragueDawley rats. Carcinogenesis. 8: 1149-1153. 1987. 